\title{
Perspectivas
}

ISSN-L: 2215-4728

DOI: http://dx.doi.org/10.15359/rp.22.5

http://www.revistas.una.ac.cr/perspectivas

N. ${ }^{\circ} 22$. Enero-Junio, $2021 ・$ pp. 1-17

\section{Sebastián Plá en primera persona: Diálogo sobre teorías y epistemologías en la enseñanza de la historia}

Sebastián Plá in the First Person: Dialogue on Theories and Epistemologies in the Teaching of History

\author{
Sebastián Plá Pérez
}

Fecha de recepción: 15/03/2021

Resumen: En esta entrevista se conversa con el profesor Sebastián Plá sobre su trayectoria profesional y los trazos epistemológicos de su pensamiento tanto de la pedagogía en general y la enseñanza de la historia en particular. En cuanto a la enseñanza de la historia el profesor Plá habla sobre las dimensiones del conocimiento histórico escolar, el papel de las narrativas históricas y la interculturalidad en el currículo y la necesidad de repensar la investigación educativa desde formas más horizontales en la producción de conocimiento.

\begin{abstract}
In this interview, a conversation is held with Professor Sebastián Plá about his professional career and the epistemological traits of his thinking about pedagogy and specifically history teaching. Regarding the teaching of history, Professor Plá talks about the dimensions of school historical knowledge, the relations between historical narratives and interculturality on curriculum the school curriculum and the need to rethink educational research to reach a more democratic knowledge.
\end{abstract}

* Mexicano. Doctor en Pedagogía por la Universidad Nacional Autónoma de México (UNAM), Ciudad de México, México. Profesor e investigador en el Instituto de Investigaciones sobre la Universidad y la Educación (IISUE), Universidad Nacional Autónoma de México (UNAM), Ciudad de México, México. Correo electrónico: sebastianpla@gmail.com. ORCID: https://orcid.org/0000-0001-8613-1607 
Palabras claves: Estudios Sociales; pedagogía; historia; currículo; epistemología.
Keywords: Social Studies; pedagogy; history; curriculum; epistemology.

\section{Entrevistadores: \\ Jéssica Ramírez Achoy ${ }^{1}$ \\ David González Sánchez ${ }^{2}$}

\section{Presentación}

Sebastián Plá es Doctor en Pedagogía por la Universidad Autónoma de México, actualmente se desempeña como investigador educativo, y se ha especializado en enseñanza de la historia desde el pensar históricamente. También ha trabajado el discurso curricular. Entre sus obras más destacadas están: Calidad educativa. Historia de una política para la desigualdad (2018), Saberes sociales para la justicia social: educación y escuela en América Latina (2017), La investigación en enseñanza de la historia en América Latina (2014). En el nivel regional es uno de los autores más reconocidos por los aportes para discutir y pensar la enseñanza de la historia, más allá de las fronteras de la historia y la pedagogía. Sus aportes actuales se dirigen hacia la justicia curricular.

Jéssica: En tus cuentas de redes sociales afirmas ser un pedagogo en contradicción. ¿Qué significa esta idea para Sebastián Plá?

Sebastián: Importante para mí fue definirme como pedagogo, en primer lugar; en segundo, siempre en contradicción, porque no vengo directamente de la pedagogía como disciplina (aunque tengo un

1 Costarricense. Doctora en Didáctica por la Universidad Tecnológica de Pereira (UTP), Pereira, Risaralda, Colombia. Académica en la Escuela de Historia de la Universidad Nacional (UNA), Sede Central Omar Dengo, Heredia, Costa Rica.

Correo electrónico: jessica.ramirez.achoy@una.cr.

ORCID: https://orcid.org/0000-0002-1795-3006.

2 Costarricense. Máster en Estudios Latinoamericanos por el Instituto de Estudios Latinoamericanos (IDELA), Universidad Nacional (UNA), Sede Central Omar Dengo, Heredia, Costa Rica. Profesor en la Universidad Estatal a Distancia (UNED), San José, Costa Rica. Correo electrónico: dagonzalez@uned.ac.cr. ORCID: https://orcid.org/0000-0002-4820-2803. 
doctorado en pedagogía). Y, en tercer lugar, hay que entenderlo en el contexto de las redes sociales, en este caso Twitter (que no soy demasiado tuitero, no tengo la capacidad de otros de estar tanto tiempo ahí). Además, las redes sociales tienen un carácter performativo, por lo que se asumen de una u otra manera personajes. En este caso mi Twitter es básicamente profesional. En ese sentido, es un carácter performativo de representar lo que soy, pero también en la construcción del personaje ¿Es falso lo que digo sobre ser un pedagogo en contradicción? No, claramente no es falso lo que digo en Twitter, soy yo el que lo pone, pero lo pongo en lenguaje de Twitter, lo pongo en el contexto de las redes sociales y en el contexto del carácter performativo que tiene toda red social.

Ahora ¿porque pedagogía y pedagogo? Yo estudié historia. Siempre tuve mucho gusto por la teoría de la historia. Y por el conocimiento sobre el pasado, disfrutaba leer las obras de Braudel o leer sobre Mesoamérica, historia antigua o Medioevo, leía a George Duby, y a la vez, me concentraba mucho en Michel de Certeu, Paul Veyne o Paul Ricoeur y me gustaban las historias, las grandes historias. Pero no se me dio la investigación histórica como tal, el archivo no fue mi fuerte, reconozco la importancia del archivo y no fue lo mío.

De joven empecé a trabajar como docente, vinculado a la literatura en formación de lectores. Y ahí desarrollé el gusto de ser profesor, pero conservaba la fuerza y la presencia de la disciplina histórica. Por eso comencé a estudiar investigación educativa. Poco a poco me fui conformando en investigador educativo. Con el paso de los años, comprendí que la pedagogía, entendida en su tradición más filosófica y humanística, se enfrentaba directamente a la investigación educativa, especialmente a partir de los años noventa del siglo XX, cuando la investigación educativa anglosajona cooptó la investigación internacional y mexicana.

En esa disyuntiva, me di cuenta de que cierta pedagogía abría la posibilidad de estar en un mundo liminal, intermedio, por un lado tenía el pensamiento científico, pero por otro, pensar la realidad a partir de elementos filosóficos, artísticos y culturales que me permitieron comprender el campo de la enseñanza de la historia.

Hoy trabajo en análisis político del discurso, a partir de mi formación en un seminario de investigación de la doctora Rosa Nidia Buenfil, del Departamento de Investigaciones Educativas de México. También 
mi profesor Alfredo Furlán, que es pedagogo, me enseñó elementos de la pedagogía fundamentales para mí. Por eso, yo me siento en contradicción en cuanto fue importante definirme como pedagogo, como alguien que está interesado en lo educativo, pero en contradicción en esta lucha entre cierta historia que quiere ser ciencia, cierta sociología que impacta mucho en la investigación educativa, ciertas formas de producción de la investigación educativa y todo el lado humanístico, filosófico y literario, que están ahí luchando en mi interior. Hasta el momento mi carrera ha sido del lado más disciplinar, más científica, pero ahora quiero impulsarme más hacia el lado humanístico, pensar la educación desde la educación y ahí es donde entraría la enseñanza de las ciencias sociales.

Jéssica: Ahora que hablas sobre estos caminos académicos que te han llevado a donde estás hoy con las investigaciones que hacés ¿cómo llegaste al pensamiento histórico?, el cual ha sido uno de los grandes aportes que has hecho en América Latina con la publicación de tu tesis de maestría en 2005.

Sebastián: Valoro mucho la experiencia y la historia personal. Tuve la fortuna de terminar mi carrera de historia y entrar a trabajar en un proyecto nacional que se llamaba los Libros del rincón, ${ }^{3}$ que fue un proyecto muy innovador en el principio de los años noventa, y hoy es la base de las bibliotecas de aula en el sistema educativo mexicano. Yo no sabía nada de educación pero me volví docente de talleres de promoción de la lectura para maestros en educación primaria. En este proyecto conocí parte de la realidad del sistema educativo mexicano, ahí me di cuenta de que me gustaba la docencia y el mundo educativo. También estaba mi gusto por la historia y vinculé estos elementos: por un lado, la experiencia con la enseñanza de la lectoescritura, de la promoción lectora en educación primaria y con maestros y, por otro lado, mi gusto por la historia. La experiencia fue muy importante porque en realidad yo llegué a la enseñanza de la historia por la promoción de la lectura. Me di cuenta de lo que hacen los maestros en sectores muy diversos del

3 Proyecto de la Secretaría de Educación Pública de México que fomenta la lectura en espacios escolares. 
país, fuera en la Ciudad de México o en estados del sur y del norte de la república. Aprendí a valorar el esfuerzo de los profesores y la importancia de ser docente.

Con un poco de estudio, noté que la enseñanza de la historia se había desarrollado de manera embrionaria en México con investigadoras como Mireya Lamoneda o Luz Elena Galván, quienes habían hecho esfuerzos para construir algunos principios didácticos. Sin embargo, para mí la investigación más relevante de esa época fue la de Silvia Ayala de la Universidad de Guadalajara. Ella realizó un estudio de corte etnográfico en el aula sobre los saberes sociales. A mí me pareció que era el más importante porque se realizó en una preparatoria (colegio) marginal de Guadalajara y hablaba de cómo interactuaban los chicos con el saber histórico. Creo que es uno de los libros más importantes que se produjo en los noventa sobre la enseñanza de la historia y todavía tiene mucha vigencia, a pesar de que se lee muy poco. Es una investigación seria que me influyó mucho.

Por un lado, estaba la experiencia en campo, y por otro, mi gusto por la teoría, una fuerte influencia del giro narrativo en la historiografía, especialmente de Hayden White y el contenido de la forma. El carácter cultural de la producción histórica de Michel de Certeau me marcó porque en la Universidad que yo estudié era el historiador que se seguía, aunque también era amigo de mi padre. Además, me interesó el giro narrativo de Paul Ricoeur.

Tuve la influencia del constructivismo que venía ya desde los noventa, especialmente de Mario Carretero y luego de Joan Pagès. Pero a mí no me gustaba el carácter cognitivo, me parece que el caso de Carretero es muy sólido desde la ciencia educativa y, en particular, de la psicología educativa, pero no es exactamente lo que me gusta, porque yo leía a Foucault y a Derridá y no entendía cómo Carretero podía ser tan esquemático en los temas de la escuela. Entonces empecé a estudiar un poco investigación educativa y tuve la influencia de otra gran corriente que es la de conceptualización del saber. En ese momento leí sobre la transposición didáctica, en didáctica de las matemáticas, y me pareció un texto brillante. Hay una visión más cultural que la interpreta como la producción de un saber específico. Y una más disciplinar, que es cómo se lleva el saber sabio, o sea el disciplinar universitario, al saber 
enseñado en el aula, pero hay tantos procesos culturales que ya no es el mismo conocimiento. Entonces, entre que yo traía una corriente de la historia que trataba de deconstruir la visión relativamente positiva de la ciencia, y que yo escribía más del lado teórico me cuestioné: ¿qué es el conocimiento histórico? ¿Es una narración? ¿Es otra forma de contar? Y me di cuenta de que estaba influido por el pensamiento posestructuralista que fracturó la visión monolítica del conocimiento histórico en particular y de las ciencias sociales en general.

Entonces estudié investigaciones que decían: la cultura escolar se construye de manera independiente, hay prácticas que son básicamente escolares, hay partes que son exclusivas de la escuela y no solo la idea de lo nacional, porque la idea nacional, por supuesto, está en la enseñanza de la historia muy marcada, pero en muchas cosas más: hay moral, hay luchas políticas, olvidos, exclusiones que se disputan la escuela. Y eso me hizo que lo tratara de conjuntar y me dio por resultado el pensar históricamente, en parte de la forma en que lo han trabajado los anglosajones, que, sin lugar a dudas, es una forma cultural e histórica de pensar de los historiadores, pero sobre cuando el conocimiento histórico llega a la escuela, transmuta su epistemología y se convierte en otro tipo de saber histórico.

Las corrientes contemporáneas de la historiografía son muy importantes, pero el saber escolar ni siquiera sigue esa lógica. Yo no lo mido en cuál es mejor o peor, lo mido en que es una distinta forma de pensar porque están en juego otros aspectos que me llevan a pensar en el uso público de la historia y no en la ciencia histórica. Esta idea, la desarrollé en un trabajo escolar muy serio sobre la escritura de la historia en el bachillerato4, donde la influencia es, básicamente, de Michel de Certeu.

Con el paso de los años, mientras continuaba mis investigaciones, me pregunté: ¿qué es nuestro objeto de investigación? y escribí un artículo que afortunadamente ha generado mucho interés. ${ }^{5}$ Ahí expongo esa idea, de que la historia en la escuela es otra cosa que la historia profesional,

4 La obra referida es: Plá, S. (2005). Aprender a pensar históricamente. La escritura de la historia en el bachillerato. PYV editores.

5 La obra referida es: Plá, S. (2012). La enseñanza de la historia como objeto de investigación. Secuencia, (84), 163-184. 
que son otra cosa, otro saber. Y esa visión de que no es lo mismo, no le ha gustado a mucha gente. Pero a otros que vienen de la Public History, de los usos públicos de la historia, sí lo entienden, les parece un poco más lógico y fundamentado. Así es como definí mi propia forma teórica, porque siempre creí que la historia es literatura, por supuesto científica, es una narración verdadera, pero una narración al fin y al cabo que es un conocimiento creativo, genial, pero también mutante. Y cuando está en otro contexto, con otras finalidades como es la cultura escolar y el proceso de descontextualización del saber que sucede en la escuela, se produce otro conocimiento. Pero este conocimiento no es sólo una mutación del saber científico, sino es también la incorporación de muchos saberes históricos que no provienen de la ciencia histórica.

Jéssica: Y ahora que nombras el artículo sobre el objeto de estudio de la investigación en enseñanza de la historia que publicaste en 2012, allí dices que la enseñanza de la historia es una construcción de significados sobre el pasado dentro de la escuela que pueden marcarse dentro de los usos públicos de la historia del presente. Esta noción se aleja de las propuestas más disciplinares y cognitivistas, la cual es esta trayectoria más intelectual, más de construcción de Sebastián Plá a la hora de plasmar este tipo de definiciones ¿Cómo Sebastián Plá construye su propia forma de ver la enseñanza de la historia?

Sebastián: ¿Por qué pienso que enseñanza la historia es un uso público a diferencia de la mayoría de los autores? La respuesta viene de muchos lados y no lo tengo muy claro, pero voy a tratar de hacer un esfuerzo. Hay aspectos democráticos. Creo que todo el mundo puede hacer con la historia lo que quiere, incluso nada. Por supuesto, hay cosas que son falsas y hay que desmentirlas. Pero hay formas de sentir y de pensar que a veces me hacen cuestionar si la historia escolar o profesional son las mejores respuestas para ello. Al mismo tiempo, me llama la atención el carácter hiperespecializado en el historiador o la historiadora, y me molesta su identidad a veces elitista. Aquí lo que entra en juego es discutir quien es dueño de la historia y preguntarse: ¿tiene dueño?

Otro elemento a considerar es la dimensión política de la enseñanza de la historia. Aquí predomina el análisis del discurso nacionalista, 
que tiende a sólo mirar este lado y, por tanto, caer en reduccionismos simplistas. Por ejemplo, la dicotomía entre la visión romántica y la ilustrada, como lo presenta Carretero en Documento de Identidad. Pero me parece que este autor no se interesa justamente por todo el proceso cultural, social y de antagonismo inherente, por lo que yo retomo la influencia de la doctora Buenfil y en especial los trabajos de Chantal Mouffe.

Esta idea de lo político y la política me llama la atención, sobre todo en el carácter inherente, antagónico que existe en toda sociedad. Esa es una dimensión política importante, porque reconoce que hay elementos antagónicos irreductibles y si aceptamos ese antagonismo, rechazaremos la idea del consenso total, pacífico y armónico del liberalismo que ha impactado a mucha de la investigación en enseñanza de la historia. Entonces me pregunto ¿Qué es ese antagonismo y dónde está ? No solo en los debates políticos y en la historia profesional, sino en lo que Habermas llama el uso público de la historia. Por ejemplo, en torno a los libros de texto y más reciente en México cuando el presidente López Obrador solicita a los españoles disculpas por los crímenes contra los pueblos indígenas. Cuando se debate el tema en México, incluso grandes historiadores, dicen que lo importante es la restitución y la justicia, no tanto el perdón con los pueblos indígenas contemporáneos. Pero hay otros grupos que piensan lo contrario. Entonces en estos antagonismos, en estas luchas públicas por el pasado ¿a cuál se parece más la enseñanza de la historia: a la historia profesional o al uso público de la historia? Pues a la de uso público. No se trata de cómo debe ser la enseñanza de la historia, porque siempre va a estar en falta. Se trata de reconocer su característica de conocimiento, comprender la epistemología de esa otra historia que es su uso público. Comprenderlo, permitirá tener una historia democrática, colectiva, presente, dentro de la escuela.

Incluso creo que si le quitas a la historia ese carácter antagónico y político, probablemente ya no tenga sentido que se enseñe en la escuela. La mirada científica, sin lugar a dudas importante, es insuficiente. Por ejemplo, una vez una estudiante de doctorado me dijo que iba a investigar cómo la enseñanza de la historia desarrolla el pensamiento crítico ¿Por qué el pensamiento crítico? ¿Por qué por principio se piensa que por saber historia o pensar como historiador hace que pienses críticamente? 
En dado caso, la historia ha demostrado que no ha desarrollado el pensamiento crítico dentro de la escuela, ha hecho exactamente lo contrario. Por tanto ¿Qué es lo crítico de la historia? Desde la ciencia, su carácter analítico, entendida entonces la crítica en una perspectiva kantiana. Pero en el sentido político, la historia no necesariamente ha sido especialmente crítica. No es crítica en sí misma, depende del uso que se haga de ella. En la escuela, puede ser crítica, pero no lo ha sido.

Este carácter antagónico, político, esta búsqueda de la epistemología de lo que se produce en la enseñanza de la historia, no se puede entender sin la escuela. Por ejemplo, la estructura de los libros de texto, especialmente hoy en día, no hay quien los pueda leer si no es en la escuela. Son un espanto, llenas de actividades y recuadros, sin narración histórica alguna. Muchos de las de los ejercicios que hacemos en la escuela podrán estar basados en Peter Seixas o Jörn Rüsen, pero en realidad están condicionados o quizá determinados por la cultura escolar. Todo esto me lo dio la experiencia de trabajar con maestros y luego ser maestro.

Mi libro de 2005 muestra cómo los estudiantes están escribiéndome a mí como profesor. No es pensamiento histórico y ¿por qué? Porque yo soy su único interlocutor, porque hay toda una estructura histórica añeja del lector único en la escuela, en particular, a partir de secundaria. Entonces ¿Qué pasa con la historia? ¿Qué aporta la historia ciencia a la historia escolar y cómo forma parte de ella? Hoy, por ejemplo, pensaba ¿En qué momento de la enseñanza de la historia, decidimos que era más importante enseñar el cambio y la permanencia, que la explotación de los humanos por los humanos? ¿En qué momento decidimos que era más importante trabajar causa y consecuencia, que la violencia del hombre contra la mujer? Esas preguntas solo pueden tener sentido en cuanto nos ubicamos en el lado educativo de la enseñanza de la historia, no en el lado histórico.

Ahora, si me pregunto: ¿qué es historia y para qué le sirve a este chico y esta chica que no van a ser historiadores, que no van a volver a agarrar un libro de historia, que van a trabajar en los cafetales, que van a ser guías de turista o que cuidarán la flora y la fauna en el parque Manuel Antonio? Yo pensaría más bien en una historia crítica, una historia que les enseñe, por ejemplo, a defender sus derechos laborales o que les 
enseñe a pensar críticamente desde la perspectiva política, es decir, no en el sentido de que vamos a analizar una fuente histórica, que es lo que predomina en la actualidad. Aquí es donde tengo una diferencia importante con muchos investigadores contemporáneos.

Hoy cada vez más la enseñanza de la historia se enseña a partir de la fuente primaria. No digo que no sea importante, pero creo que la enseñanza de la historia es mucho más rica a partir de las fuentes secundarias, en especial buenas narraciones escritas por historiadores o literatos. Sin embargo, hay algo paradójico en las perspectivas contemporáneas de la enseñanza de la historia. Se quiere que los estudiantes piensen como historiadores a través del análisis de fuentes, pero no se les da a leer historiadores, no se les muestra qué es una buena narración histórica. ¿Cómo van a pensar como un historiador si no leen historiadores? ¿Sólo a partir de fuentes primarias?

La paradoja todavía es más profunda. Si estamos a favor de que los estudiantes sólo lean fuentes primarias y se omite al historiador, de facto, estamos negando una función social fundamental del historiador: interpretar y contar historias sobre el pasado. Ahora bien, yo me pregunto: ¿qué escogemos leer los adultos si queremos conocer la historia de la humanidad: un libro con documentos, por ejemplo, un compilación con el Código de Hammurabi, Tucídides, una estela maya del clásico, una nota del gobierno británico contra el bloqueo continental, el documento fundacional de la ONU y la constitución sudafricana o leer una buena narración, digamos, como las de Harari? Yo me inclino por la segunda, por eso prefiero que las y los niños y jóvenes lean buenas historias y no documentos originales. Mi propuesta no excluye las fuentes, sólo las desestima como algo importante de la enseñanza de la historia. Si pensamos desde la historia, quizá lo mejor sean los documentos primarios, pero si pensamos desde lo educativo, es mejor la narración.

Ellos dicen, vamos a formar ciudadanos críticos, vamos a analizar la fuente primaria. Está bien, se puede trabajar con la fuente primaria. Pero mejor que lean un buen ensayo, una buena novela, les va a dar mejores aprendizajes. Hoy parece que la función social del conocimiento histórico es que aprendamos a pensar como los historiadores y no contarnos historias del pasado. 
David González: Vos planteás, además, la idea de la investigación en la enseñanza de la historia como un campo fronterizo. Podrías profundizar en esa idea.

Sebastián: Voy a recuperar a un autor que políticamente es muy cuestionable, pero es un autor literario extraordinario: Peter Handke. Él maneja la idea del umbral. En el umbral estás entrando pero también estás saliendo. En este sentido me refiero a lo fronterizo, lo liminal. Quizá las propuestas de los estudios culturales, por ejemplo, romper fronteras a través de lo transdisciplinario, lo multidisciplinario, lo interdisciplinario o todas juntas sería representativo de lo que es una buena investigación contemporánea. Pero en ese tiempo usé fronterizo porque estaba muy clara la idea de la historia como disciplina y la psicología como dominante en el campo educativo. Había una psicologización impresionante del campo educativo.

Yo creo que hay que regresar a la pedagogía; aunque la sociología me ofrece muchísimo. Me cuesta más la psicología porque la siento muy esquemática, muy universalizante $\mathrm{y}$, a veces, poco comprensiva de lo que es la escuela; en cambio, la sociología y la antropología no. La escuela tiene muchos elementos vitales. Entonces, el carácter fronterizo es eso, pensar la educación con la sociología, con la historia, algunos dirán con la psicología, pero otros dirán con la antropología. La historia en sí misma, y lo que voy a decir es muy obvio, pero la historia en sí misma no sabe estudiar la enseñanza de la historia, no puede porque no es su objeto de estudio. Tiene demasiado presente.

La historia puede dar elementos teóricos para estudiar la enseñanza de la historia, pero no se basta a sí misma. Lo mismo pasa con otras disciplinas. Si tú ves bien la enseñanza de la historia, no puedes quedarte en la psicología porque también el conocimiento que tú estás transmitiendo, que estás enseñando y te están pidiendo, juega un papel importante.

Si tienes una perspectiva psicológica, necesitan un poco o un mucho más bien, de historia. El conocimiento sobre el pasado no es una excusa. La psicologización de los años noventa y principios del siglo XXI propuso que lo importante es desarrollar habilidades, el conocimiento es una excusa para desarrollar las habilidades básicas. Lo que llamé en mi libro sobre calidad educativa, supracognición o un conjunto 
de habilidades universales que se han impuesto en todos los sistemas educativos. Pero no, no es lo mismo enseñar la conquista de México, la matanza de Tlatelolco o la explotación ¿Cómo puede ser una matanza una excusa? Debería ser el tema en sí mismo. Ahí es donde se volvió más importante enseñar una habilidad de lectura, por ejemplo con la fuente primaria, que enseñar la explotación, que enseñar la injusticia, que enseñar la violencia de género, que denunciar un genocidio. Es al revés, lo importante es el contenido, la habilidad es consecuencia de un buen trabajo con el contenido.

Lo que digo básicamente es que no se puede estudiar la enseñanza de la historia desde una sola disciplina, es insuficiente, porque es educativa no historiográfica y al ser educativa requieres tomar muchos elementos de procedencias muy diversas. En mi caso, creo que no podemos soslayar la filosofía, la sociología, los estudios culturales, la antropología y la pedagogía y por supuesto, la historia misma. En ese sentido es fronterizo. Pero fronterizo, porque también yo estaba en un momento donde identificaba las disciplinas separadas. Entonces, la enseñanza de la historia estaba en la frontera entre esas disciplinas. Hoy pensaría que es más una amalgama. Una concepción transdisciplinar quizá sea lo más correcto. Pero también interdisciplinar.

David: Has mencionado varias cosas importantes desde la experiencia con docentes, y creo que también en buena parte de los escritos hablás de cierto antagonismo que se genera entre el especialista en educación o entre los especialistas en general y el lugar que se le asigna al saber docente. ¿Hasta qué punto puede una investigación educativa ser un medio para introducir los saberes docentes en el orden del discurso académico y disputar la hegemonía del especialista? ¿Puede hablar el docente también dentro de este mercado académico?

Sebastián: Yo digo que no, hoy en día. Porque la investigación educativa está en la lógica del especialista. Y hoy la investigación educativa ha sido parte importante de la exclusión de las voces docentes. 
Lean al historiador Antonio Novoa. Algunos casos latinoamericanos los trabajo en mi libro sobre Calidad Educativa. ${ }^{6}$

En una investigación, que no he terminado, sobre la conformación del experto, considero que antiguamente el experto era el maestro, pero a este se le arrebata la palabra y ahora se le dice que los que saben de educación son otros expertos. Por ejemplo, en los sistemas evaluativos nacionales, los expertos saben de evaluación pero no de docencia, pero son los evaluadores quienes definen qué es una docencia idónea y qué no.

Yo creo que en la enseñanza de la historia, como la estamos trabajando en América Latina, cada vez está más encaminada a arrebatarle la palabra a los docentes. La experiencia docente es fundamental, pero también hay una confusión. A mí también me dicen: "tú eres el experto, el académico y no conoces la experiencia del aula”. Bueno, que yo no he estado en tú aula, no significa que no pueda conocer la escuela y el aula. El conocimiento de la experiencia es fundamental, pero también se puede conocer mucho más allá de la experiencia. Y creo que hay muchas posiciones expertas que niegan el saber docente. Un caso opuesto, siempre en diálogo, fue el profesor Joan Pagès.

El problema es buscar un equilibrio: valorar la experiencia docente, pero también que los profesores se den cuenta que hay mucho más que eso, muchas formas más de pensar la enseñanza y la escuela. El investigador necesita cambiar ciertas prácticas, pues ocupa el saber docente. Por esto, hay que trabajar de manera conjunta. Yo creo que nuestra función como investigadores es producir conocimientos, pero sobre todo tratar de ayudar al profesor o la profesora a pensar un poco de manera distinta o, por lo menos, que vea que hay otras formas de pensar la práctica.

La investigación puede servir desde ciertas perspectivas horizontales, donde se investiga en conjunto con profesores, no de modo extractivista donde se toman datos de los sujetos y se publica para el reconocimiento académico. Los investigadores pueden hacer mucho con y por el docente, pero no necesariamente ha sido así.

6 La obra referida es: Plá, S. (2018). Calidad educativa. Historia de una política para la desigualdad. IISUE. 
David: En algunas ocasiones se habla de enseñar desde donde está situado quien habla y entonces te preguntamos: ¿Qué significa para ti pensar la enseñanza de la historia desde América Latina?

Sebastián: Latinoamérica es mi casa, mi lengua, es mi forma de pensar. Yo nací en Uruguay y tengo nacionalidad mexicana. Tuve la fortuna de estar en Costa Rica y Guatemala, he tenido la posibilidad de trabajar en Chile, en Argentina, en Brasil, en Uruguay y a distancia en Colombia; yo mismo he ido a Bolivia, a Perú, Cuba. Mis padres fueron exiliados políticos. Yo crecí en una casa donde América Latina era una. Por supuesto, con sus múltiples visiones y por eso todos mis estudios tienen una dimensión internacional. América Latina está ahí. Ahora bien ¿cuál es el contexto latinoamericano y qué es lo que estamos produciendo de pensamiento latinoamericano para poder pensar la enseñanza de la historia desde Latinoamérica?

Esta pregunta puede tener una influencia decolonial y tratar de emanciparnos en lo que podamos del capitalismo cognitivo o más bien del colonialismo cognitivo. Tenemos demasiada dependencia académica. Todos los autores que cité, la mayoría son europeos y yo mismo tengo que liberarme. Ahora, yo creo que en la enseñanza de la historia hay una influencia fuerte de España. Es una dependencia epistemológica muy importante.

Las investigaciones en didáctica de la historia tienen mucho potencial para trabajar en contextos complejos e importantes, pero el contexto debería ser texto, parte sustancial de lo que investigamos y decimos. Es contradictorio, nuestra América dentro del texto se convierte en contexto, pues narramos lo que pasa aquí con las palabras, los métodos y los significados de lo que se produjo en otros contextos. Pero ojo, no estoy diciendo que tengamos que negar lo que se produce en Europa o Norteamérica, por el contrario, debemos dialogar con ellos pero ubicados en otro lugar, en otro posicionamiento discursivo y en otro locus de enunciación político.

Un ejemplo, el concepto de ciudadanía es de una ciudadanía liberal básicamente de modo europeo. Está bien, no digo que no tenga potencialidades el concepto de ciudadanía, pero está ausente la ciudadanía práctica del movimiento de los Sin Tierra, de los Zapatistas, de lo 
que investigadoras mexicanas han llamado ciudadanos inesperados, es decir, formas de hacer política por fuera de los marcos liberales, muy característicos de nuestra región. Pero como no están dentro del marco liberal, son ilegítimos. Otro ejemplo, con el movimiento feminista que hay en México, ¿qué enseñanza de la historia tenemos que pensar? ¿Cómo leer las fuentes de los cronistas del XVI con modelos creados para contextos canadienses o pensar históricamente para luchas contra los feminicidios? El modelo de Seixas es excelente, debemos conocerlo, ¿pero responde a los desplazados por la violencia armada, a la desigualdad económica, a la migración que caracteriza a nuestra región?

Yo creo que en Latinoamérica tenemos que hacer un esfuerzo todavía mayor por crear nuestros discursos, a pesar de que hay varios campos como las teorías decoloniales, las teorías de la dependencia o la teología de la liberación. La enseñanza de la historia se sigue profesionalizando notablemente pero nos falta mucha teoría y es la teoría las que nos permitirá hablar desde aquí, desde la Amazonía, el Petén o los Andes. Hay que hacer un libro de teoría de la historia general, aunque por suerte pronto se publicará uno, en Brasil, coordinado por Juliana Alves de Andrade y Nilton Mullet Pereira.

David: Particularmente en tu ensayo, Las 5 narrativas interculturales, propones unos tipos de narrativas y dices: "una verdadera justicia curricular para todos, incluidos los más desfavorecidos, por supuesto, es que todos tengan derecho a conocer nuestra historia desde nuestra perspectiva cultural. Y, sobre todo, que aprendamos a pensar la historia desde diferentes perspectivas culturales." Tomando tu trayectoria y el énfasis que le dabas a lo político: ¿Se desliza un poco hacia lo cultural y eso denota una inflexión decolonial en tus ideas?

Sebastián: Sí y no. Lo cultural es político también. Entendido lo político en el sentido de Mouffe. Lo político es el carácter inherentemente antagónico de la sociedad. Yo he estado investigando mucho sobre la educación, la justicia social y lo intercultural. La interculturalidad por razones diversas, pero, básicamente, porque me lo enseñaron unas alumnas mías. Y justicia social porque ya he empezado a escribir bastante con cosas no directamente vinculadas con la enseñanza de la 
historia, me ha interesado el problema de la justicia curricular. La justicia curricular, que llegó a América Latina por la influencia de Jurjo Torres, pero que en realidad es un concepto de Raewyn Connell de los años 90 . Este autor dice que los contenidos escolares tienen que, en primer lugar, atender a los más desfavorecidos. Con esta idea, tomé la filosofía política de de Nancy Fraser. Me interesó mucho su idea tripartita de reconocimiento, redistribución y representación.

Entonces me puse a leer más sobre la justicia social y la relación entre paridad participativa, redistribución y reconocimiento. O sea, puedes reconocer a los indígenas, pero si no les devuelves la tierra no pasa nada, no hay justicia social alguna. Si no hay redistribución, pues no hay justicia, no basta con el reconocimiento. Fraser sostiene, además, que hay diferentes tipos de acciones para la búsqueda de la paridad participativa, el reconocimiento a la diversidad cultural e identitaria y la redistribución de la riqueza. Están son las acciones transformativas o las acciones positivas, las dos buscan diferentes formas de justicia social. La acción positiva es aquella que busca dar las habilidades a los sujetos para que puedan competir en igualdad de condiciones y tener éxito en la sociedad desigual. Una discriminación positiva es un ejemplo claro.

La acción transformativa es aquella que no busca que el sujeto triunfe en la sociedad desigual, sino la deconstrucción y el desmembramiento de las propias condiciones que producen la desigualdad. En ese sentido, me parece que la enseñanza de la historia es una enorme productora de desigualdad, en cuanto elemento simbólico y que su potencialidad para la justicia curricular está en la transformación de los aspectos simbólicos que subyacen a la naturalización de las desigualdades de diversa índole dentro de la sociedad.

Hay un texto que publiqué en Brasil. Es un ensayo en forma de diálogo. Trata de dos personas discutiendo estas posiciones de justicia social y su relación con la enseñanza de la historia. Ahí me di cuenta que pensar históricamente se inclina hacia la acción positiva. Y cierta teoría de pedagogía crítica y de otras como las teorías decoloniales van más hacia la transformativa. Las dos buscan la justicia social, son diferentes caminos. Incluso, puede haber casos en que ambos tipos de acciones se complementen. 
Después, continuado mis estudios sobre la justicia curricular, y con cuestionamientos producidos por investigaciones sobre educación intercultural de colegas como Ana Laura Gallardo, Carlo Rosa o Marion Lloyd, me exigí repensar el problema cultural de la enseñanza de la historia. Para mí, es un giro que va hacia lo cultural pero que no pierde su dimensión política. Todo esto tiene que ver quizá, con mi propio gusto por escuchar otras formas de narrar las historias.

Hay una novela que me marcó: Los Trazos de la Canción de Bruce Chatwin. El autor es un escritor inglés que murió muy joven. El libro habla de un grupo que va haciendo mapas mientras se traslada y canta. Su forma de cartografía es el canto y la trashumancia, porque la novela es sobre lo que para Chatwin es la memoria original nómada de los seres humanos. Es el canto de la trashumancia humana. Hay otra película que me influyó en esta perspectiva hace décadas: "Donde sueñan las hormigas verdes" de Werner Herzog, de Australia. Estas obras me mostraron que hay narrativas históricas excluidas de la escuela, y que se pueden estudiar desde formas culturales diversas, como la literatura y el mito. Yo creo que la gran habilidad que debería enseñar la enseñanza de la historia es la narración, la narración no ficcional. No tanto la lectura de fuentes primarias, sino la narración. Leer y escribir narraciones. Sobre el tema de diferentes formas culturales de narrar la historia y su enseñanza, puede verse en un capítulo mío en el que coordiné con Sandra Patricia Rodríguez Ávila, Saberes sociales para la justicia social: educación y escuela en América Latina ${ }^{7}$.

David y Jéssica: Muchas gracias por el tiempo, la entrevista y las buenas ideas. Sin duda, este diálogo nos ha permitido conocer mejor a Sebastián Plá como pedagogo e investigador educativo.

7 La referencia aludida es: Plá, S., y Ávila, S. (Eds.). (2017). Saberes sociales para la justicia social: Educación y escuela en América Latina. Universidad Pedagógica Nacional. 